\title{
Sacrocolpopexy using autologous rectus fascia: cohort study of long-term outcomes and complications
}

\author{
Rui Wang ${ }^{1}$, Krista Reagan ${ }^{2}$, Sarah Boyd ${ }^{3}$, and Paul Tulikangas ${ }^{1}$ \\ ${ }^{1}$ Hartford Hospital \\ ${ }^{2}$ MultiCare Health System \\ ${ }^{3}$ Penn State Health Milton S Hershey Medical Center
}

September 24, 2021

\begin{abstract}
Objective: To evaluate objective and subjective outcomes of patients who underwent sacrocolpopexy using autologous rectus fascia to provide more data regarding non-mesh alternatives in pelvic organ prolapse surgery. Design: Cohort study with retrospective and prospective data. Setting: A single academic medical center. Population: Women who underwent abdominal sacrocolpopexy using autologous rectus fascia between January 2010 and December 2019 Methods: Patients were recruited for a follow-up visit including completing the Pelvic Floor Distress Inventory (PFDI) and Pelvic Organ Prolapse Quantification (POPQ) exam. Demographic and clinical characteristics were collected. Main Outcome Measures: Composite failure, anatomic failure, symptomatic failure, and retreatment. Results: During the study period, 132 women underwent sacrocolpopexy using autologous rectus fascia. Median follow-up time was 2.2 years. Survival analysis showed that composite failure was $0.8 \%$ (CI $0.1-5.9 \%$ ) at 12 months, $3.5 \%$ (CI 1.1-10.7\%) at 2 years, $13.2 \%$ (CI $7.0-24.3 \%$ ) at 3 years, and $28.3 \%$ (CI $17.0-44.8 \%)$ at 5 years. Anatomic failure was $0 \%$ at 12 months, $1.4 \%$ (CI $0.2-9.2 \%$ ) at 2 years, $3.1 \%$ (CI $0.8-12.0 \%$ ) at 3 years, and $6.8 \%$ (CI $2.0-22.0 \%$ ) at 5 years. Symptomatic failure rate was $0 \%$ at 12 months, $1.3 \%$ (CI $0.2-9.0 \%$ ) at 2 years, $2.9 \%$ (CI $0.7-11.3 \%$ ) at 3 years, and $13.1 \%$ (CI $5.3-30.3 \%$ ) at 5 years. Retreatment rate was $0.8 \%$ (CI $0.1-5.9 \%$ ) at 12 months and 2 years, 9.4\% (CI $4.2-20.3 \%$ ) at 3 years, and $13.0 \%$ (CI $6.0-27.2 \%)$ at 5 years. Conclusions: Autologous rectus fascia sacrocolpopexy may be considered a safe and effective alternative for patients who desire to avoid synthetic mesh.
\end{abstract}

\section{Sacrocolpopexy using autologous rectus fascia: cohort study of long-term outcomes and com- plications}

Rui Wang, MD ${ }^{1}$, Krista Reagan, $\mathrm{MD}^{2}$, Sarah Boyd, $\mathrm{MD}^{3}$, Paul Tulikangas, MD ${ }^{1}$

${ }^{1}$ Department of Obstetrics and Gynecology, Division of Female Pelvic Medicine and Reconstructive Surgery, Hartford Hospital, Hartford, CT

2 Department of Urogynecology and Pelvic Reconstructive Surgery, MultiCare Health System, Tacoma, WA

${ }^{3}$ Division of Female Pelvic Medicine and Reconstructive Surgery, Penn State Health Milton S. Hershey Medical Center, Hershey, PA

\section{Corresponding author}

Rui Wang

85 Seymour Street, Suite 525

Hartford, CT 06106

860-972-1908 
Rui.Wang@hhchealth.org

\section{Conflict of interest}

The authors report no conflict of interest.

Short Title: Autologous rectus fascia sacrocolpopexy

Word count

Abstract: 248

Main text: 2,648

\section{Abstract}

Objective: To evaluate objective and subjective outcomes of patients who underwent sacrocolpopexy using autologous rectus fascia to provide more data regarding non-mesh alternatives in pelvic organ prolapse surgery.

Design: Cohort study with retrospective and prospective data.

Setting: A single academic medical center.

Population: Women who underwent abdominal sacrocolpopexy using autologous rectus fascia between January 2010 and December 2019

Methods: Patients were recruited for a follow-up visit including completing the Pelvic Floor Distress Inventory (PFDI) and Pelvic Organ Prolapse Quantification (POPQ) exam. Demographic and clinical characteristics were collected.

Main Outcome Measures: Composite failure, anatomic failure, symptomatic failure, and retreatment.

Results: During the study period, 132 women underwent sacrocolpopexy using autologous rectus fascia. Median follow-up time was 2.2 years. Survival analysis showed that composite failure was $0.8 \%$ (CI 0.1-5.9\%) at 12 months, $3.5 \%$ (CI 1.1-10.7\%) at 2 years, $13.2 \%$ (CI $7.0-24.3 \%$ ) at 3 years, and $28.3 \%$ (CI $17.0-44.8 \%$ ) at 5 years. Anatomic failure was $0 \%$ at 12 months, $1.4 \%$ (CI $0.2-9.2 \%$ ) at 2 years, $3.1 \%$ (CI $0.8-12.0 \%$ ) at 3 years, and $6.8 \%$ (CI 2.0-22.0\%) at 5 years. Symptomatic failure rate was $0 \%$ at 12 months, $1.3 \%$ (CI $0.2-9.0 \%$ ) at 2 years, $2.9 \%$ (CI $0.7-11.3 \%$ ) at 3 years, and $13.1 \%$ (CI $5.3-30.3 \%$ ) at 5 years. Retreatment rate was $0.8 \%$ (CI $0.1-5.9 \%$ ) at 12 months and 2 years, $9.4 \%$ (CI $4.2-20.3 \%$ ) at 3 years, and $13.0 \%$ (CI $6.0-27.2 \%$ ) at 5 years.

Conclusions: Autologous rectus fascia sacrocolpopexy may be considered a safe and effective alternative for patients who desire to avoid synthetic mesh.

Funding : internal departmental research funding

Tweetable abstract: Sacrocolpopexy using autologous rectus fascia is a safe and effective alternative to synthetic mesh.

Keywords: autologous fascia, complications, sacrocolpopexy, surgical outcomes

\section{INTRODUCTION}

Pelvic organ prolapse is a common condition. Each year, over 300,000 surgeries are performed in the U.S. for pelvic organ prolapse. ${ }^{1,2}$ Abdominal sacrocolpopexy is an effective technique with several studies demonstrating long-term effectiveness. ${ }^{3}$ Sacrocolpopexy is most often performed using a synthetic mesh to suspend the vaginal apex to the anterior longitudinal ligament of the sacrum. However, there is a risk of mesh-related complications associated with this procedure. The Colpopexy and Urinary Reduction Efforts (CARE) trial showed an estimated rate of mesh complications of $10.5 \% .^{4}$ 
Prior studies using alternatives to mesh for sacrocolpopexy have had mixed results. In a randomized trial that evaluated the use of a porcine dermal xenograft versus synthetic mesh, durability was similar at 1 year. ${ }^{5}$ The porcine graft is no longer commercially available. In another randomized trial of sacrocolpopexy using cadaveric fascia lata versus synthetic mesh, synthetic mesh resulted in better outcomes at 5 years. ${ }^{6}$

Given patient and provider concerns surrounding mesh use in pelvic reconstructive surgeries, more data is needed regarding non-mesh alternatives. Abdominal sacrocolpopexy using autologous rectus fascia may provide an effective and safe alternative to sacrocolpopexy using synthetic mesh. Although case series have been published with patients undergoing autologous rectus fascia sacrocolpopexies, ${ }^{7,8}$ no large cohort studies have been conducted with long-term follow-up. The aim of this study is to evaluate objective and subjective outcomes of patients who underwent sacrocolpopexy using autologous rectus fascia.

\section{METHODS}

\section{Study design}

This study was reviewed and approved by the Hartford Hospital Institutional Review Board. Our cohort study included all women who underwent abdominal sacrocolpopexy using autologous rectus fascia between January 2010 and December 2019 at a single academic medical center. While we most commonly performed minimally invasive sacrocolpopexy using synthetic mesh, patients were offered a non-mesh alternative using autologous tissue. Patients were thoroughly counseling regarding limited available data on the outcomes of this procedure. Patients were eligible for the study if they were female, [?]18 years old and underwent abdominal sacrocolpopexy using autologous rectus fascia. Patients were excluded if they had a history of a prior sacrocolpopexy or if synthetic mesh was used for the procedure. Eligible patients were identified by review of operative log books and billing records, and surgical procedures were confirmed by review of the full operative notes. As part of standard practice, all patients were asked preoperatively to complete a validated prolapse symptom questionnaire (Pelvic Floor Dysfunction Inventory, PFDI-20) and this data was also collected. Pre- and post-operative pelvic organ quantification (POP-Q) measurements were recorded.

Patients were recruited for a follow-up visit including completing the PFDI-20 and POP-Q exam. Over the course of 2015 to 2020, eligible patients were called and offered to return to the office for an examination and complete the follow-up PFDI questionnaire and if unable or unwilling to come for a visit, were asked to complete the questionnaire by phone. Examinations were performed by an author not involved in the initial surgery. For patients who did not return to the office for a study visit, their most recent POP-Q examination from routine postop follow-up was used. Follow-up time was defined as the time between surgery and the date of the last post-operative POP-Q examination or PFDI-20 questionnaire.

Our primary outcomes were subjective symptoms as measured by PFDI-20 and anatomic prolapse staging as measured by POP-Q examinations. We reported a composite measure of treatment failure that included retreatment for prolapse (pessary or surgery), anatomic outcomes as defined by any POP-Q measure beyond the hymen, and symptomatic outcome defined as a positive response (with any degree of bother other than "not at all") to the PFDI question "Do you usually have a bulge or something falling out that you can see or feel in your vaginal area?". Secondary outcomes included post-operative complications including readmission, transfusion, reoperation and infections. Demographic and medical data included age, race, ethnicity, body mass index, previous hysterectomy, prior incontinence surgery, prior prolapse surgery, smoking status, parity, history of diabetes mellitus, hypertension, hyperlipidemia, and asthma. Surgical data included concomitant surgeries, operative time, postoperative hematocrit level, length of stay, and postoperative complications within 30 days.

\section{Analysis}

Descriptive statistics were calculated with the use of standard methods for means, medians, and proportions. Categorical data were analyzed using chi-square or Fisher's exact test where appropriate. Continuous variables were analyzed using paired or one-sample t-tests where appropriate. Statistical analysis was performed with Stata software (version 12; StataCorp, College Station, TX). All analyses were considered statistically 
significant at p-values $<0.05$.

\section{Surgical technique}

All procedures were performed through a low transverse or low vertical incision to harvest a strip of rectus fascia approximately $10 \mathrm{~cm}$ in length by $2 \mathrm{~cm}$ in width. The rectus fascia was either attached to the anterior vaginal wall or reconstructed in $\mathrm{Y}$ configuration and secured to the anterior and posterior vaginal walls with delayed absorbable or permanent sutures depending on surgeon preference. The apex of the rectus fascia strip was attached to the anterior longitudinal ligament at the sacrum with permanent sutures. The peritoneum was closed over the exposed length of rectus fascia.

\section{RESULTS}

There were a total of 132 patients who underwent sacrocolpopexy using autologous rectus fascia during the study period. Patient characteristics are shown on Table 1. Mean age was 59.0 (range 32-83) years. Mean body mass index was $25.9 \mathrm{~kg} / \mathrm{m}^{2}\left(\mathrm{SD} 3.8 \mathrm{~kg} / \mathrm{m}^{2}\right.$ ). Preoperatively, $23.7 \%$ of patients presented with stage II prolapse, $71.0 \%$ with stage III prolapse and $4.6 \%$ with stage IV prolapse. This was the initial prolapse repair for $75.8 \%$ of the patients. Concomitant surgeries performed included: total hysterectomy $(47.7 \%)$, supracervical hysterectomy (11.4\%), Burch colposuspension (61.4\%), posterior colporrhaphy (50.8\%). Mean operative time was 258 minutes (SD 104). The mean post-operative hematocrit was $33.0 \mathrm{~g} / \mathrm{dL}$ (SD 3.1 $\mathrm{g} / \mathrm{dL}$ ). Two patients (1.5\%) received blood transfusion. Mean length of stay was 2.2 days (SD 1.1 days). The length of stay was significantly lower for patients who underwent surgery after the implementation of Enhanced Recovery After Surgery (ERAS) protocol compared to before ERAS (1.8 \pm 0.6 days vs. $2.4 \pm 1.3$ days, respectively; $\mathrm{P}=0.008)$.

Out of all 132 eligible patients, 95 (72\%) consented to have additional follow up for the purposes of this study. For patients who were not able to have additional follow up during the study period, follow up data were collected from chart review. A total of $128(97 \%)$ of patients had postoperative POPQ exams either as part of routine care or follow up during the study.

Median follow-up time was 2.2 years, ranging from 1 month to 6.2 years. Survival analyses showed that the probability of composite failure was $0.8 \%$ (CI $0.1-5.9 \%$ ) at 12 months, $3.5 \%$ (CI $1.1-10.7 \%$ ) at 2 years, $13.2 \%$ (CI $7.0-24.3 \%$ ) at 3 years, and $28.3 \%$ (CI $17.0-44.8 \%$ ) at 5 years (Figure 1). The probability of anatomic failure was $0 \%$ at 12 months, $1.4 \%$ (CI $0.2-9.2 \%$ ) at 2 years, $3.1 \%$ (CI $0.8-12.0 \%$ ) at 3 years, and $6.8 \%$ (CI $2.0-22.0 \%$ ) at 5 years (Figure 2). The probability of symptomatic failure was $0 \%$ at 12 months, $1.3 \%$ (CI $0.2-9.0 \%$ ) at 2 years, $2.9 \%$ (CI $0.7-11.3 \%$ ) at 3 years, and $13.1 \%$ (CI $5.3-30.3 \%$ ) at 5 years (Figure 3 ). The probability of retreatment was $0.8 \%$ (CI $0.1-5.9 \%$ ) at 12 months and 2 years, 9.4\% (CI $4.2-20.3 \%$ ) at 3 years, and $13.0 \%$ (CI $6.0-27.2 \%$ ) at 5 years (Figure 4). A total of 7 patients underwent surgical retreatment for prolapse, 3 patients had an anterior and posterior colporrhaphy, 2 patients had only a posterior colporrhaphy, 1 patient had posterior colporrhaphy with a sacrospinous ligament fixation, and 1 patient had an anterior and posterior colporrhaphy with a sacrospinous ligament fixation.

On follow-up, $7.7 \%$ of patients had stage $0,63.1 \%$ had stage I, $28.5 \%$ had stage II, and $0.8 \%$ had stage III prolapse. None had stage IV prolapse. All POP-Q measurements except for total vaginal length were significantly improved from baseline $(\mathrm{P}<0.001)$. In terms of subjective outcomes, PFDI scores were significantly improved from baseline (99.8, CI 86.7-112.9) to follow-up (52.3, CI 42.0-62.6; P<0.001).

In terms of post-operative complications, $13.6 \%$ of patients were treated for post-operative infections other than urinary tract infections. Of these, $6.1 \%$ of patients had surgical site infections and $3.8 \%$ had pneumonia. Rate of readmission within 30 days of surgery was $9.1 \%$. This included $5.3 \%$ of patients readmitted with ileus or small bowel obstruction (0.8\% requiring surgical intervention), and $1.5 \%$ of patients readmitted for pain. There were no patients with post-operative venous thromboembolism and no other patient requiring reoperation within 30 days.

Postoperative voiding dysfunction was present in $4.5 \%$ of patients beyond the immediate postoperative period. The rate of overactive bladder symptoms was $35.6 \%$ post-operatively and $12.9 \%$ received medications. 
Although $32.6 \%$ of all patients reported stress urinary incontinence symptoms post-operatively, $10.6 \%$ had positive cough stress test, and $13.6 \%$ underwent subsequent treatment for stress urinary incontinence. The rate of subsequent mid-urethral slings was $5.3 \%$, the rate of subsequent pubovaginal slings was $3.8 \%$, and the rate of subsequent urethral bulking was $7.6 \%$, with $3.0 \%$ of patients undergoing both urethral bulking and mid-urethral slings. Overall $19.7 \%$ of patients received physical therapy post-operatively. No patients were diagnosed or treated for incision site hernia.

\section{DISCUSSION}

\section{Main Findings}

We showed in a cohort of patients that sacrocolpopexy using autologous rectus fascia was an effective treatment for pelvic organ prolapse with subjective and objective outcomes that were sustained for up to three years. PFDI scores remained decreased by almost half for patients with over three years of followup. Over $63 \%$ of patients had stage I or less prolapse on exam at last follow-up. And only $5 \%$ of patients underwent reoperation for prolapse recurrence. In addition, the use of autologous fascia eliminated the up to $10.5 \%$ mesh complications noted in the CARE trial follow-up. ${ }^{4}$

\section{Interpretation}

The use of mesh for pelvic reconstructive surgery has come under intense scrutiny over the last decade. Mesh complications have become an increasing concern for patients. In 2008, the Food and Drug Administration (FDA) issued a Public Health Notification regarding the adverse events related to the use of surgical mesh in urogynecology. ${ }^{2}$ In 2011, the FDA issued an update noting that serious complications associated with surgical mesh for transvaginal repair of prolapse is not rare. ${ }^{2}$ Subsequently, in 2012, the FDA issued orders to conduct post-market surveillance studies to address the safety and effectiveness of transvaginal mesh prolapse repairs. In 2016, surgical mesh for transvaginal prolapse repair was reclassified from Class II to Class III. In 2017, all urogynecologic mesh was reclassified from Class I to Class II. Finally, in 2019, vaginal mesh kits were removed from the market. ${ }^{9}$ Although this did not include mesh used for sacrocolpopexy and mid-urethral slings, studies have shown that the rates of prolapse and incontinence surgeries using mesh have decreased during this time. ${ }^{10}$

A recent review of the available literature on autologous fascia prolapse repairs showed that there have been only six case series. ${ }^{7}$ Several series reported on the use of pedicled rectus fascia flaps. ${ }^{11-13}$ One retrospective study that included free rectus fascia graft compared outcomes between mesh, Pelvicol xenograft, and autologous fascia for abdominal sacrocolpopexy but only had 15 patients in the autologous fascia group. ${ }^{8}$ The authors concluded that recurrence was more likely with a Pelvicol graft but was equivalent for mesh and autologous graft. Oliver et al. reported a series of 19 patients with excision of sacrocolpopexy mesh with replacement of a free rectus fascia graft. ${ }^{14}$ Seth et al. reported a case series of 7 patients who underwent autologous rectus fascia sacrocolpopexy and sacrohysteropexy with free Y grafts. Mean follow-up was 16 months with all patients having durable results at last follow-up. ${ }^{7}$ Another alternative to mesh sacrocolpopexy is allograft fascia. However, there have been studies that reported significant early failure rates with sacrocolpopexy using allograft fascia. ${ }^{15,16}$ Antigenicity, immune response, and post-harvest preservation techniques are all factors that affect graft remodeling and thus the success and function of an allograft, but that do not negatively affect autologous fascia grafts.

This cohort provided subjective and objective outcome data on an alternative to mesh sacrocolpopexy. To our knowledge, this was the largest cohort available with long-term follow-up. Our low retreatment rate for prolapse was consistent with those noted in studies using mesh graft. Our postoperative ileus or SBO rate was comparable to slightly lower than that seen in the 2 year data from the CARE trial (5.3\% vs $6.8 \%$ ). In addition, the use of this autologous graft eliminated the $10.5 \%$ complication rate of mesh erosion noted in the CARE trial. ${ }^{4}$ One concern with harvesting autologous rectus fascia is the risk of incisional hernias. In the SISTer trial, hernias occurred in $1.2 \%$ of patients who underwent a rectus fascia pubovaginal sling for treatment of incontinence. ${ }^{17}$ There were no cases of incisional hernias in our cohort. Mean length of stay in our cohort was 2.2 days and this is a notable disadvantage of the procedure compared to minimally invasive 
sacrocolpopexy with mesh grafts with a mean length of stay less than 24 hours. ${ }^{18}$ Prolonged length of stay has been associated with increased hospital-acquired infections, decreased mobility and longer postoperative recovery time. ${ }^{19,20}$ As such, there has been a trend across all surgical specialties to develop protocols and techniques that aid in shortening length of stay. ${ }^{21,22}$ The ongoing COVID-19 pandemic has further pushed this goal to decrease hospital exposures and led to same-day discharge after pelvic reconstructive surgery with good outcomes. ${ }^{23}$ The advent of ERAS programs have aided in this goal. We did see a decrease in length of stay in patients that underwent surgery after standardized ERAS protocols were initiated at our hospital.

\section{Strengths and Limitations}

The strengths of our study include the prospective collection of primary outcomes, the size of our population, and the duration of follow-up. We included both subjective and objective outcome measures as either type of outcome measure alone may not capture the patient's true experience and quality of life after pelvic reconstructive surgery. PFDI-20 is a validated measure of patient's pelvic floor symptoms, which we were able to compare across time for each patient. We present the largest cohort of patients who underwent sacrocolpopexy using autologous rectus fascia in the literature and our results provide valuable information for providers and patients seeking alternatives to mesh sacrocolpopexy. We were able to recruit patients for follow-up assessment up to over 6 years from the time of surgery, which allowed us to analyze the outcomes for patients who had at least 3 years of follow-up.

Our results are limited by the fact that some data are collected retrospectively, the range of follow-up time, and single institution data. Certain demographic and medical history data were collected retrospectively and may be limited by the accuracy of medical records. Patients chose their surgical treatment after extensive counseling and there is likely to be selection bias. However, our study is targeted at this group of patients, who did not desire mesh material in their prolapse surgery, in order to provide long-term outcomes data for a non-mesh alternative to sacrocolpopexy. Due to the wide range of years during which this procedure was performed and in order to collect a large cohort of patients, some patients did not have long-term follow-up available. We were able to analyze the subgroup of patients who had long-term follow-up, which demonstrated similar outcomes and supported our main results. Because this study was conducted at a single institution, our patient population may not be generalizable to other regions or countries.

Although our results showed that sacrocolpopexy using autologous rectus fascia resulted in significant prolapse improvements and low rates of retreatment, our cohort was subject to selection bias as described. Our findings support future research utilizing randomized controlled trials to provide more robust evidence for sacrocolpopexy using autologous rectus fascia for patients who desire a non-mesh alternative.

\section{CONCLUSION}

Sacrocolpopexy using autologous rectus fascia may be considered a safe and effective alternative to synthetic mesh. Our results showed significant improvements in objective and subjective outcomes that were sustained over several years. Our results contribute significantly to the evidence in the literature regarding autologous rectus fascia as an alternative to mesh sacrocolpopexy.

\section{Contribution to Authorship:}

RW: data collection, data analysis, manuscript writing, manuscript editing, final approval

KR: study design, data collection, manuscript writing, manuscript editing, final approval

SB: data collection, manuscript editing, final approval

PT: study design, manuscript editing, final approval

\section{Funding:}

This study was funded through internal division research funds. 


\section{Institutional Review:}

This study was reviewed and approved by the Hartford Hospital Institutional Review Board. Initial approval date: 08/25/2014. Last approval date: 04/24/2020.

\section{Acknowledgements}

The authors are thankful for the assistance in accommodating study patients for office visits by the Hartford Hospital Urogynecology office staff, and especially for Colleen Mellen, APRN, for her contribution to performing a portion of the study visits.

References

1. Smith F, Holman C, Moorin R, Tsokos N. Lifetime risk of undergoing surgery for pelvic organ prolapse. Obs Gynecol . 2010;116:1096-1100.

2. US Food and Drug Administration. Urogynecologic Surgical Mesh: Update on the Safety and Effectiveness of Transvaginal Placement for Pelvic Organ Prolapse .; 2011. https://www.fda.gov/media/81123/download.

3. Siddiqui N, Grimes C, Casiano E, et al. Mesh sacrocolpopexy compared with native tissue vaginal repair: a systematic review and meta-analysis. Society of Gynecologic Surgeons Systematic Review Group.Obs Gynecol . 2015;125:44-55.

4. Nygaard I, Brubaker L, Zyczynski H, et al. Long-term outcomes following abdominal sacrocolpopexy for pelvic organ prolapse.JAMA . 2013;309(19):2016-2024.

5. Culligan P, Salamon C, Priestley J, Shariati A. Porcine dermis compared with polypropylene mesh for laparoscopic sacrocolpopexy: a randomized controlled trial. Obs Gynecol . 2013;121(1):143-151.

6. Tate S, Blackwell L, Lorenz D, Steptoe M, Culligan P. Randomized trial of fascia lata and polypropylene mesh for abdominal sacrocolpopexy: 5-year follow-up. Int Urogynecol $J$. 2011;22(2):137-143.

7. Seth J, Toia B, Ecclestone H, et al. The autologous rectus fascia sheath sacrocolpopexy and sacrohysteropexy, a mesh free alternative in patients with recurrent uterine and vault prolapse: A contemporary series and literature review. 2019:193-197. doi:10.4103/UA.UA

8. Quiroz LH, Gutman RE, Shippey S, et al. Abdominal sacrocolpopexy: anatomic outcomes and complications with Pelvicol, autologous and synthetic graft materials. Am J Obs Gynecol . 2020;198(5):557.e1-5.

9. U.S. Food \& Drug Administration. Urogynecologic Surgical Mesh Implants. https://www.fda.gov/medicaldevices/implants-and-prosthetics/urogynecologic-surgical-mesh-implants. Published 2019. Accessed October 20, 2020.

10. Winkelman W, Modest A, Richardson M. U.S. Food and Drug Administration Statements About Transvaginal Mesh and Changes in Apical Prolapse Surgery. Obs Gynecol . 2019;134(4):745-752.

11. Yaqub U, Shahzad N. Uterine suspension by rectus sheath flap, in selected cases of UV prolapse. PJMHS . 2013;7:322-333.

12. Jenkins D, McCoubrie S. Vault prolapse: a new approach. Aust N Z J Surg . 1992;62:805-808.

13. Mahendru R. An effective and safe innovation for the management of vault prolapse. Ann Surg Innov Res . 2010;4:6.

14. Oliver J, Chaundhry Z, Medendorp A, et al. Complete excision of sacrocolpopexy mesh with autologous fascia sacrocolpopexy. Urology . 2017;106:65-69.

15. Huang Y, Lin A, Chen K, Pan C, Chang L. High failure rate using allograft fascia lata in pubovaginal sling surgery for female stress urinary incontinence. Urology . 2001;58:943-946. 
16. Fitzgerald M, Mollenhauer J, Bitterman P, Brubaker L. Functional failure of fascia lata allografts. Am J Obs Gynecol . 1999;181:1339-1345.

17. Chai TC, Albo ME, Richter HE, et al. Complications in Women Undergoing Burch Colposuspension Versus Autologous Rectus Fascia Sling for the Treatment of Stress Urinary Incontinence. 2009;181(5):21922197. doi:10.1016/j.juro.2009.01.019.Complications

18. Nosti P, Umoh Andy U, Kane S, et al. Outcomes of abdominal and minimally invasive sacrocolpopexy: a retrospective cohort study.Female Pelvic Med Reconstr Surg . 2014;20(1):33-37.

19. Rosman M, Rachminov O, Segal O, Segal G. Prolonged patients' In-Hospital Waiting Period after discharge eligibility is associated with increased risk of infection, morbidity and mortality: a retrospective cohort analysis. BMC Heal Serv Res . 2015;15:246.

20. Committee on Gynecologic Practice. ACOG Committee Opinion No. 750: Perioperative Pathways: Enhanced Recovery After Surgery. Obs Gynecol . 2018;132(3):e120-e130.

21. Varadhan K, Neal K, Dejong C, Fearon K, Ljungqvist O, Lobo D. The enhanced recovery after surgery (ERAS) pathway for patients undergoing major elective open colorectal surgery: a meta-analysis of randomized controlled trials. Clin Nutr . 2010;29:434-440.

22. Miralpeix E, Nick A, Meyer L, et al. A call for new standard of care in perioperative gynecologic oncology practice: Impact of enhanced recovery after surgery (ERAS) programs. Gynecol Oncol . 2016;141(2):371-378.

23. Berger A, Tan-Kim J, Menefee S. Comparison of 30-Day Readmission After Same-Day Compared With Next-Day Discharge in Minimally Invasive Pelvic Organ Prolapse Surgery. Obs Gynecol . 2020;135(6):13271337.

Figure 1. Kaplan-Meier curve for composite failure

Figure 2. Kaplan-Meier curve for anatomic failure

Figure 3. Kaplan-Meier curve for symptomatic failure

Figure 4. Kaplan-Meier curve for retreatment

Table 1. Characteristics of study population

Table 2. Composite failure, retreatment, anatomic failure, and symptomatic failure outcomes

Table 3. Secondary subjective and objective outcomes

Table 4. Post-operative complications ( $\mathrm{N}=132)$ 

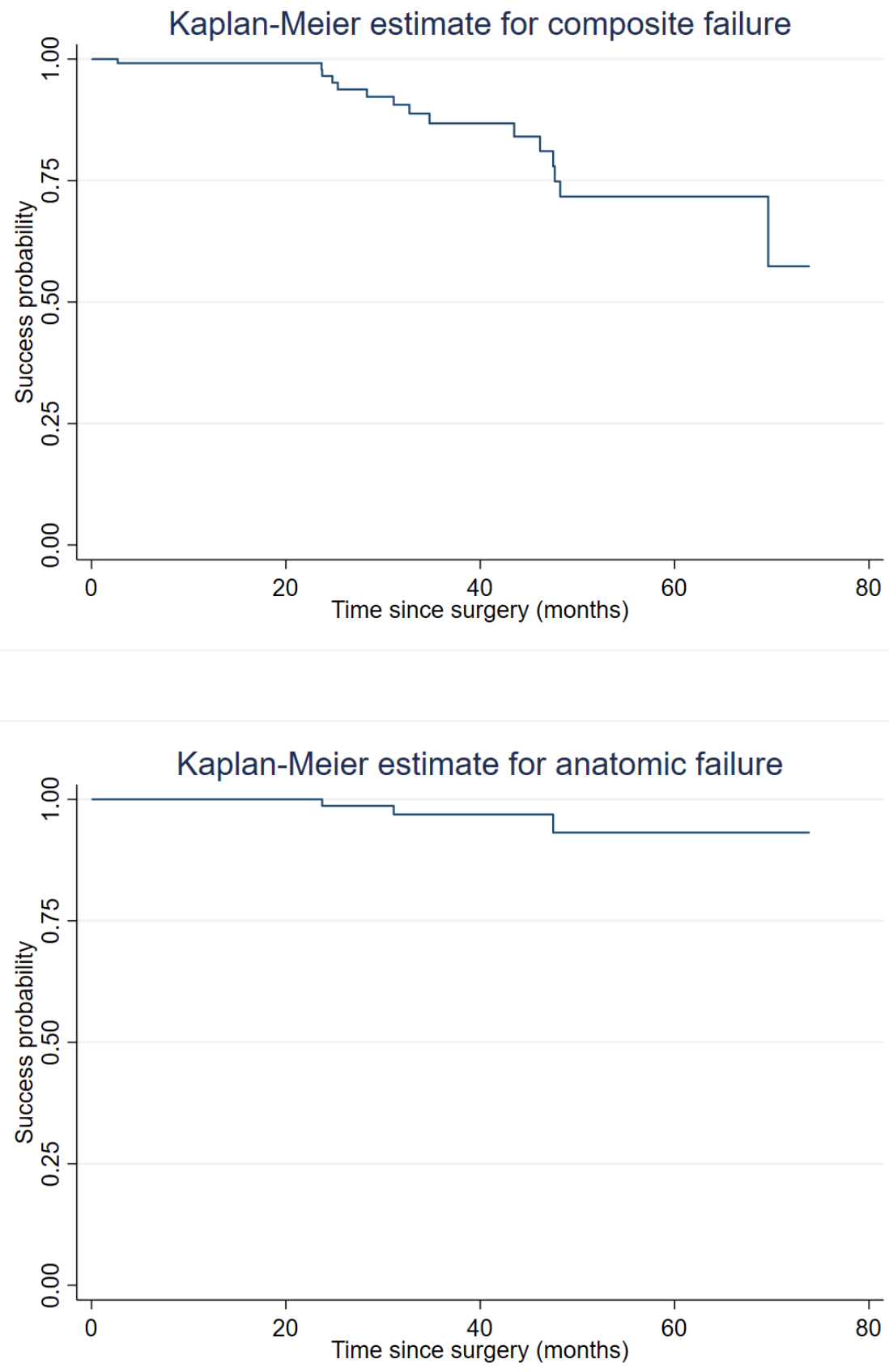

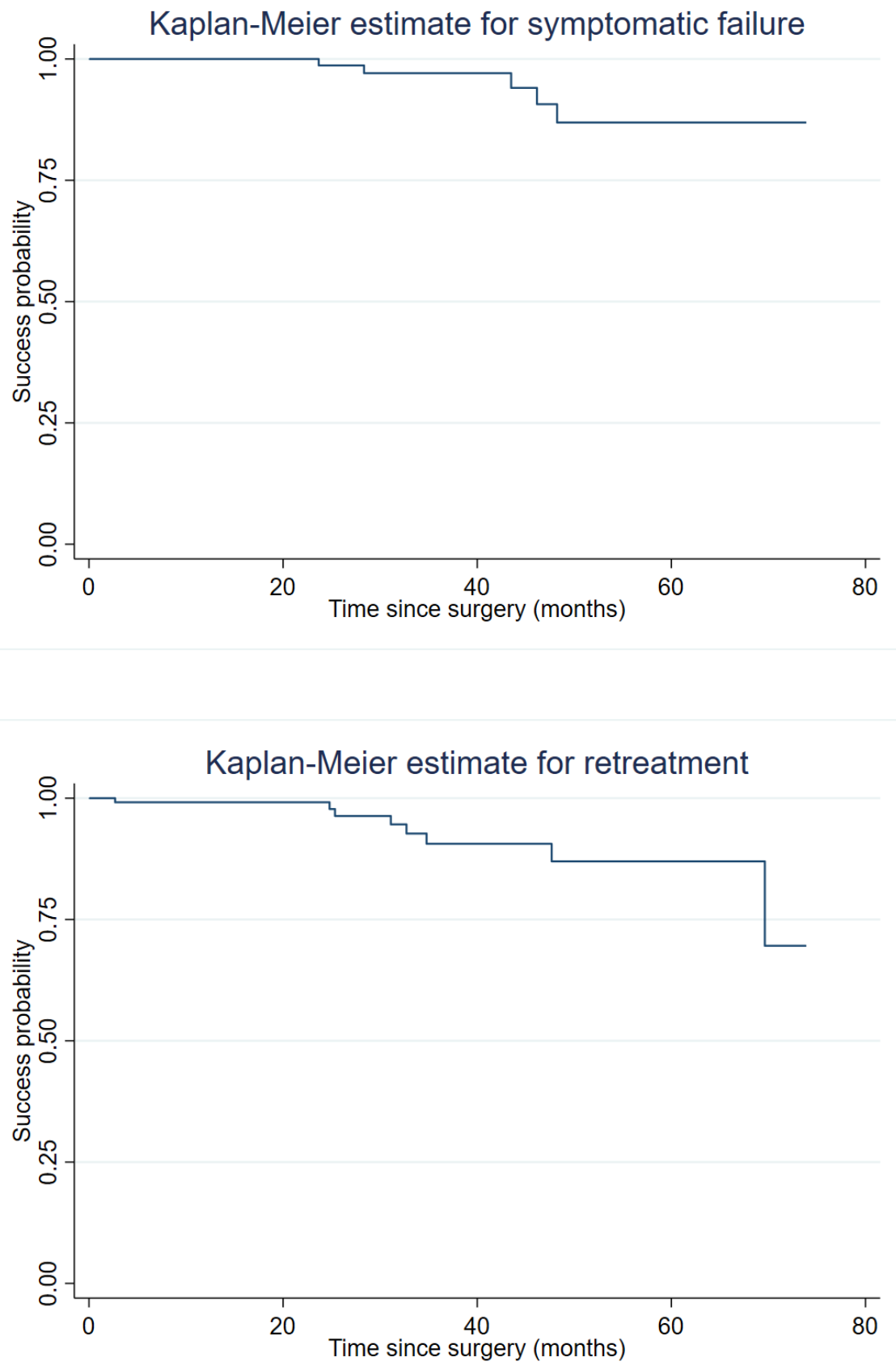

\section{Hosted file}

Table 1.docx available at https://authorea.com/users/435817/articles/538579-sacrocolpopexyusing-autologous-rectus-fascia-cohort-study-of-long-term-outcomes-and-complications

\section{Hosted file}

Table 2.docx available at https://authorea.com/users/435817/articles/538579-sacrocolpopexyusing-autologous-rectus-fascia-cohort-study-of-long-term-outcomes-and-complications 


\section{Hosted file}

Table 3.docx available at https://authorea.com/users/435817/articles/538579-sacrocolpopexyusing-autologous-rectus-fascia-cohort-study-of-long-term-outcomes-and-complications

Hosted file

Table 4.docx available at https://authorea.com/users/435817/articles/538579-sacrocolpopexyusing-autologous-rectus-fascia-cohort-study-of-long-term-outcomes-and-complications 